\title{
Expression of Oncogenic Molecules in Pediatric Ulcerative Colitis
}

\author{
Nobuyasu Arai Takahiro Kudo Kazuhide Tokita Reiko Kyodo \\ Masamichi Sato Eri Miyata Kenji Hosoi Tamaki lkuse Keisuke Jimbo \\ Yoshikazu Ohtsuka Toshiaki Shimizu
}

Department of Pediatric and Adolescent Medicine, Juntendo University Graduate School of Medicine, Tokyo, Japan

\section{Keywords}

Pediatric ulcerative colitis · Colitis-associated cancer ·

Cancer-related gene

\begin{abstract}
Introduction: Long-term disease duration of ulcerative colitis (UC) is known to increase the risk of developing colorectal cancer in adults; however, this association has not been genetically analyzed in children with UC. Herein, we examined the expression of cancer-related genes in the colonic mucosa of pediatric UC patients and their risk of developing colorectal cancer. Methods: Microarray analysis of cancerrelated gene expression was conducted on rectal mucosa biopsy specimens randomly selected from pediatric cases, including 4 active-phase UC cases, 3 remission-phase UC cases, and 3 irritable bowel syndrome control cases. The subject pool was then expanded to 10 active-phase cases, 10 remission-phase cases, and 10 controls, which were analyzed by real-time polymerase chain reaction (PCR) and immunohistochemical staining. Results: The microarray results indicated significantly higher expression levels of cancer-related genes PIM2 and SPI1 in the active group than in the
\end{abstract}

karger@karger.com www.karger.com/dig

Karger $\stackrel{\text { ' }}{5}$

BOPEN ACCESS
(C) 2021 The Author(s)

Published by S. Karger AG, Basel

This is an Open Access article licensed under the Creative Common Attribution-NonCommercial-4.0 International License (CC BY-NC) (http://www.karger.com/Services/OpenAccessLicense), applicable to the online version of the article only. Usage and distribution for commercial purposes requires written permission. remission and control groups $(p<0.05)$. Real-time PCR confirmed that PIM2 and SPI1 expression levels were significantly higher, whereas TP53 and APC expression levels were significantly lower, in the active-phase group than in the remission and control groups $(p<0.05)$. Immunohistochemical staining for PIM2, SPI1, TP53, and APC proteins supported the real-time PCR results. Conclusions: Expression levels of previously unreported cancer-related genes in adult UC patients were significantly higher in pediatric UC patients than in controls. Inflammation of the gastrointestinal mucosa increased the expression levels of cancer-related genes even in childhood-onset UC cases, suggesting that chronic inflammation from childhood may increase the risk of colorectal cancer development.

(c) 2021 The Author(s)

Published by S. Karger AG, Basel

\section{Introduction}

Inflammatory bowel disease (IBD) causes chronic inflammation in the gastrointestinal tract with cycles of relapse and remission. IBD is classified into 2 main types, ulcerative colitis (UC) and Crohn's disease, and causes symp- 
toms such as abdominal pain, diarrhea, and bloody stool. The number of patients with both diseases in Japan is increasing. Indeed, a national survey estimated 170,000 patients with UC and 40,000 with Crohn's disease in Japan in 2016 [1]. The number of pediatric patients with IBD is also increasing. Patients with UC have a higher carcinogenic risk than the general population [2]; specifically, the risk of colorectal cancer increases due to long-term morbidity associated with UC $[3,4]$. Thus, colorectal carcinogenesis is a serious concern for patients with long duration from UC onset. A recent study indicated that elderly onset of UC ( $\geq 60$ years of age at diagnosis) increases cancer risk [5], but cancer rates are also high in patients with childhood-onset UC in Europe and the USA. Prophylactic colorectal resection was proposed to reduce colorectal cancer mortality in patients diagnosed with UC $<15$ years of age, as these patients demonstrated a significantly higher risk of developing colorectal cancer than those diagnosed at an older age [6]. Similarly, a 2017 cohort study demonstrated that childhood-onset IBD increased risk of future cancer [7]. However, in childhood-onset UC, lesion development is rapid, and the clinical characteristics often differ from those in adults; that is, many patients already have severe disease at diagnosis. Furthermore, genetic factors are likely involved in young-onset cases, such as for patients in whom the disease may be caused by a congenital immune abnormality. However, no pediatric studies have analyzed the non-neoplastic mucosa and selection of patients at high risk of cancer complications, as has been performed for adults [8].

The aim of this study was to analyze the expression of cancer-related genes in childhood-onset UC patients and to elucidate changes in their expression to predict future carcinogenesis risk even in childhood. These findings could provide indications for colorectal resection in consideration of future colorectal cancer complications, which could be included in UC treatment guidelines, and help stratify risk classes from childhood, becoming a basis for establishing future surveillance guidelines.

\section{Materials and Methods}

\section{Patients and Samples}

Pediatric patients with UC were randomly selected from those who visited the Juntendo University Hospital Department of Pediatrics, Tokyo, Japan, from January 2011 to December 2018 with reported abdominal pain, diarrhea, and bloody stool. Ten patients with UC diagnosed in the active phase were classified into Group A, and 10 patients with a treatment history of UC in clinical remission were classified into Group B. Ten patients with all negative tests who received a diagnosis of IBS according to the Rome IV criteria [9] were classified into Group C. Individuals with a history of treatment for severe liver, heart, kidney, blood, or other systemic diseases were excluded. Gastrointestinal endoscopy was performed on all patients, and specimens of rectal mucosa were collected for histopathological examination. Samples of rectal mucosa were collected and analyzed based on previously described procedures $[8,10]$. This study protocol complied with the ethical guidelines of the Declaration of Helsinki and was approved by the hospital Ethics Committee of Juntendo University Hospital (No. 18-226), and written informed consent was obtained from patients and their legal guardians. Specimens were used for microarray, real-time polymerase chain reaction (PCR), and immunohistochemical (IHC) analyses.

\section{RNA Extraction from Mucosal Biopsy Samples}

Endoscopic mucosal biopsy specimens were preserved in RNA later (Applied Biosystems, Life Technologies, Carlsbad, CA, USA) and stored at $-80^{\circ} \mathrm{C}$. For microarray and real-time PCR analyses, specimens were disrupted with RNeasy lysis buffer and homogenized, and RNA was extracted using the RNeasy Mini Kit (Qiagen, Germantown, MD, USA). RNA quantity and purity were assessed using a NanoDrop ND-1000 spectrophotometer (Thermo Fisher Scientific, Waltham, MA, USA) and the Experion RNA StdSens Analysis Kit (Bio-Rad Laboratories, Hercules, CA, USA).

\section{Microarray Hybridization and Data Analysis}

Rectal mucosa biopsy samples were randomly selected from 4 active-phase patients, 3 remission-phase patients, and 3 IBS controls, and the microarray expression profiles of cancer-related genes were compared. Relative gene expression was determined by microarray analysis using the Ambion WT Expression Kit (Applied Biosystems) and the GeneChip WT Terminal Labeling and Controls Kit (Affymetrix, Santa Clara, CA, USA), according to the manufacturers' instructions. Specifically, total RNA was reverse-transcribed into cDNA using random primers. Next, cRNA was synthesized from cDNA by in vitro transcription. The cRNA was then used as the second cycle template for cDNA synthesis. The cDNA was fragmented, labeled, and hybridized to the GeneChip Human Gene 2.0 ST Array (Affymetrix) at $40^{\circ} \mathrm{C}$ for $16 \mathrm{~h}$, which contained approximately 40,000 genes. The chip was washed, stained with streptavidin-phycoerythrin, and scanned using GeneChip Scanner $30007 \mathrm{G}$ (Affymetrix). Scanned images were converted to CEL files using GeneChip Command Console Software (AGCC) (Affymetrix), and data analysis was performed using GeneSpring GX v11 software (Agilent Technologies, Santa Clara, CA, USA). Data from each chip were normalized using the RMA algorithm. Results were searched, and differentially expressed genes between Groups A, B, and $\mathrm{C}$ were selected. We also searched for genes that were previously reported to be specifically associated with colorectal cancer.

\section{Real-Time PCR Analysis}

Real-time PCR was performed using the cDNA synthesized from the colonic biopsy RNA preparation using the High-Capacity cDNA Reverse Transcription Kit (Applied Biosystems) with the 7,500 Fast Real-Time PCR System (Applied Biosystems). TaqMan gene expression assays targeting PIM2, SPI1, TP53, and APC (Thermo Fisher Scientific) were employed to examine gene expression levels in the rectal mucosa of patients with UC and IBS, which were compared with expression levels of differentially expressed genes identified in the microarray analysis. The expression level of each gene was normalized to that of glyceraldehyde 3-phosphate dehydrogenase $(G A P D H)$ using the standard curve method. 
Table 1. Subject characteristics in patients with UC (active group and inactive group) and irritable bowel syndrome (normal control, NC group)

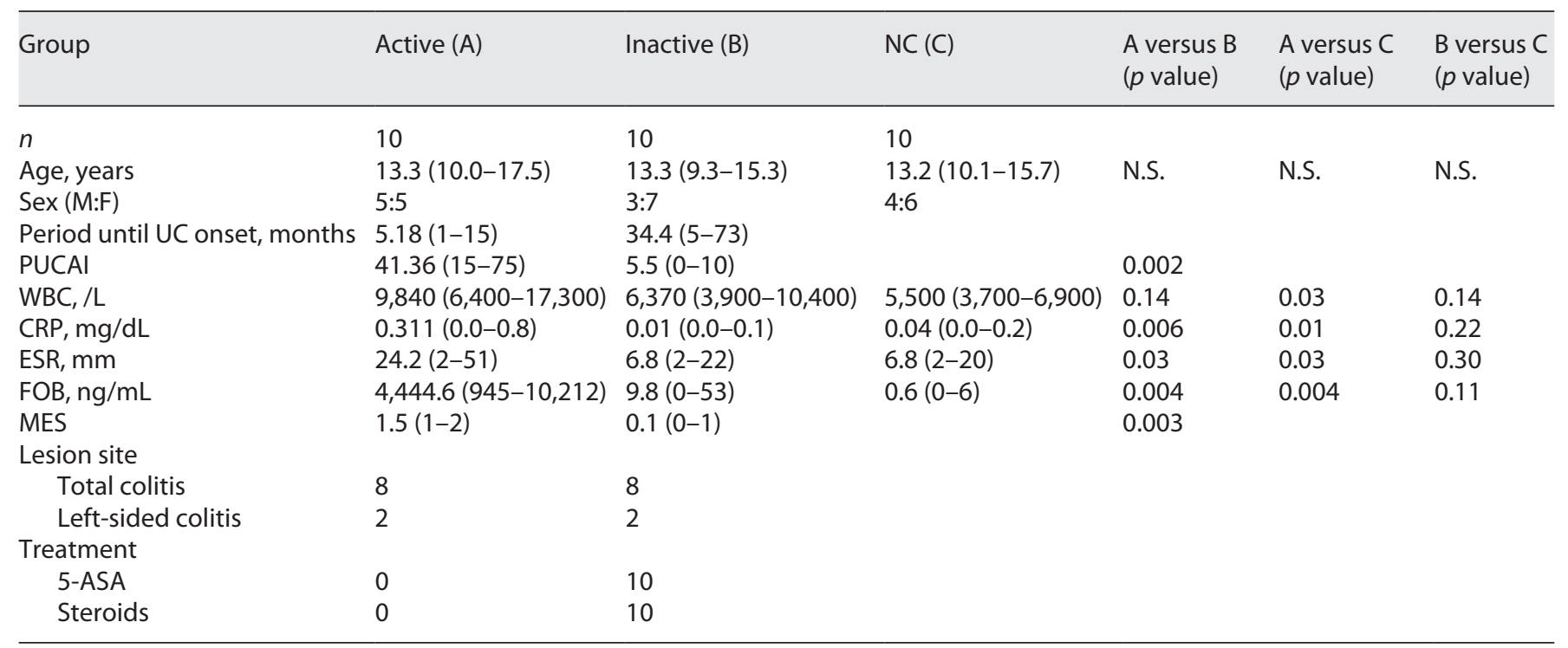

PUCAI, Pediatric Ulcerative Colitis Activity Index; 5-ASA, 5-aminosalicylic acid; WBC, white blood cell; CRP, C-reactive protein; ESR, erythrocyte sedimentation rate; FOB, fecal occult blood; MES, Mayo endoscopic subscore; UC, ulcerative colitis.

IHC Analysis of PIM2, SPI1, TP53, and APC

IHC staining of mucosal epithelium tissue specimens was performed using the Benchmark GX IHC/ISH Automated Staining Instrument and Nexes IHC Automated Immunohistochemistry Staining System (both Ventana Medical Systems, Tucson, AZ, USA) according to the manufacturer's protocols. Serial sections ( 3 $\mu \mathrm{m}$ thick) were prepared from formalin-fixed, paraffin-embedded rectal mucosa samples. After washing the paraffin sections with deparaffinized water, the following primary antibodies were diluted and added to the sections: PIM2 (1:100; Novus Biologicals, Centennial, CO, USA), SPI1 (1:100; Abcam, Cambridge, UK), TP53 (1:100; OriGene Technologies, Inc., Rockville, MD, USA), and APC (1:50; Abcam). Antigen activation was performed for 10 min at $121^{\circ} \mathrm{C}$ using $10 \mathrm{mM}$ citrate buffer ( $\mathrm{pH} \mathrm{6.0)}$. All heating was performed in an autoclave, and after washing, the sections were incubated with a biotin-conjugated secondary antibody. The sections were then incubated for 30 min with streptavidin (Dako, Carpinteria, CA, USA). Peroxidase activity was blocked with 3,3'-diaminobenzidine tetrahydrochloride containing $0.01 \%$ $\mathrm{H}_{2} \mathrm{O}_{2}$. Prior to examination by light microscopy, all sections were counterstained with hematoxylin, and normal rabbit immunoglobulin (Dako) or mouse immunoglobulin (Dako) was used as a negative control.

\section{Statistical Analysis}

Microarray data were analyzed using $t$ tests. Mann-Whitney $U$ tests were performed in patients' details and real-time PCR data. A $p$ value $<0.05$ was considered statistically significant. All statistical analyses were performed using EZR statistical software [11].

\section{Results}

Patient characteristics are listed in Table 1. There were no differences in mean age among the 3 groups. The male:female ratio was similar in Groups $\mathrm{A}$ and $\mathrm{C}$, although there were more females in Group B. The Pediatric Ulcerative Colitis Activity Index (PUCAI) [12] was higher in Group A than in Group B, whereas the mean disease duration was significantly higher in Group B than in Group A. Erythrocyte sedimentation rate (ESR) and fecal occult blood (FOB) biomarkers in Group A were significantly higher than those in Group B and Group C. The average Mayo endoscopic subscore (MES) [13] was 1.5 for Group A and 0.1 for Group B, and there was significant difference between the 2 groups. But no significant difference was observed in disease activity markers between Group B and Group C. Lesions in 16/20 patients (80\%) with UC involved the entire colon, and inflammation tended to be widespread, similar to the typical characteristics of childhood-onset UC.

\section{Microarray Gene Expression Analysis}

The results of gene microarray analysis are presented in Table 2. Exploration of the changes in cancer-related gene expression indicated that PIM2 and SPI1 expression levels were significantly higher in Group A than in Group 
Table 2. Comparison of cancer-related gene expression between groups

\begin{tabular}{|c|c|c|c|c|c|c|}
\hline NM_006875 & PIM2 & Pim2 proto-oncogene, serine/Threonine kinase & $\begin{array}{l}\text { Ratio } \\
p \text { value }\end{array}$ & $\begin{array}{l}3.22024 \\
0.01640\end{array}$ & $\begin{array}{l}3.17383 \\
0.00345\end{array}$ & $\begin{array}{l}0.98559 \\
0.78877\end{array}$ \\
\hline NM_001080547 & SPI1 & Spi-1 proto-oncogene & $\begin{array}{l}\text { Ratio } \\
p \text { value }\end{array}$ & $\begin{array}{l}1.51520 \\
0.00528\end{array}$ & $\begin{array}{l}1.77061 \\
0.00128\end{array}$ & $\begin{array}{l}1.16856 \\
0.26196\end{array}$ \\
\hline NM_000038 & $A P C$ & Adenomatous polyposis coli & $\begin{array}{l}\text { Ratio } \\
p \text { value }\end{array}$ & $\begin{array}{l}0.90633 \\
0.43101\end{array}$ & $\begin{array}{l}0.95005 \\
0.75160\end{array}$ & $\begin{array}{l}1.04824 \\
0.67150\end{array}$ \\
\hline
\end{tabular}

A, pediatric patients with active UC; B, pediatric patients with UC in remission; C, pediatric patients with irritable bowel syndrome (control). UC, ulcerative colitis.

$\mathrm{C}(p<0.05)$. However, the expression levels of these genes between Groups B and C did not differ significantly. The expression levels of TP53 and APC, genes that are reportedly associated with colorectal cancer in adults, did not differ significantly among the 3 groups.

\section{Real-Time PCR Analysis}

Real-time PCR results are shown in Figures 1 and 2. Expression levels of oncogenes PIM2 and SPI1 were significantly higher in Group A than in Group C (PIM2: $p=$ 0.002 , SPI1: $p=0.003)$. The expression level of PIM2 decreased significantly between Group A and Group B ( $p=$ 0.02 ), whereas the expression level of SPI1 did not differ between these 2 UC groups. Expression levels of tumor suppressor genes TP53 and APC were significantly lower in Group A than in Group C (TP53: $p=0.02, A P C: p=$ 0.002). However, the expression level of TP53 in Group B did not differ significantly from that in Group A. The expression level of APC in Group B was significantly higher than that in Group A, but did not differ significantly from that in Group C. In other words, oncogenes PIM2 and SPI1 and tumor suppressor genes APC and TP53 demonstrated opposite expression trends among the groups.

\section{IHC Analysis}

The IHC results are shown in Figures 3 and 4. Staining of target proteins by IHC revealed the same expression patterns observed by real-time PCR. Specifically, among mucosal epithelium inflammatory cells, the number of PIM2-positive cells was increased in Group A compared with that in Group C and was decreased in Group B compared with that in Group A. The number of SPI1-positive cells in the mucosal epithelium was increased in Group A compared with that in Group C and was also increased in Group B compared with that in Group A. Fewer TP53positive cells were observed in both Groups A and B compared to Group C. Although APC-stained cells showed a decreasing trend in Group A compared with Group C, similar findings were observed in Groups B and C.

\section{Discussion}

At present, UC-associated colitis-associated cancer is believed to be the result of inflammatory carcinogenesis in the mucous membrane $[8,14]$. General colorectal cancer develops according to the adenoma-carcinoma multistage sequence [15]. However, in colitis-associated cancer, an atypical mucosa (i.e., dysplasia) develops against the background of inflammation according to the dysplasia-carcinoma sequence, which varies according to the order in which gene mutations are expressed [16]. The proposed mechanism is that mucous membranes chronically inflamed by UC accumulate genetic abnormalities due to long-term inflammation and exposure to cytokines, gradually increasing the risk factors of carcinogenesis. However, the precise mechanism of colorectal cancer development from UC remains unresolved. Gene analysis in adult UC patients and studies investigating cancer-related genes have been frequently published, identifying genes associated with carcinogenesis of adult colitis-associated cancer [8, 14-18]. However, protein expression levels of genes of interest have not been reported in children. Therefore, this study is the first to comprehensively analyze cancer-related genes in pediatric UC patients. The results of this study suggest that chronic 
Fig. 1. Real-time PCR analysis of PIM2 and SPI1 expressions in rectal mucosal biopsy specimens. The expression level of each gene normalized to that of GAPDH is presented as a box-and-whisker plot; the central box covers the interquartile range, with the median indicated by the line within the box. The whiskers extend to the maximum and minimum values. $A$, pediatric active UC cases; B, pediatric UC cases in remission; C, pediatric irritable bowel syndrome cases (control). UC, ulcerative colitis; GAP$D H$, glyceraldehyde 3-phosphate dehydrogenase; PCR, polymerase chain reaction.
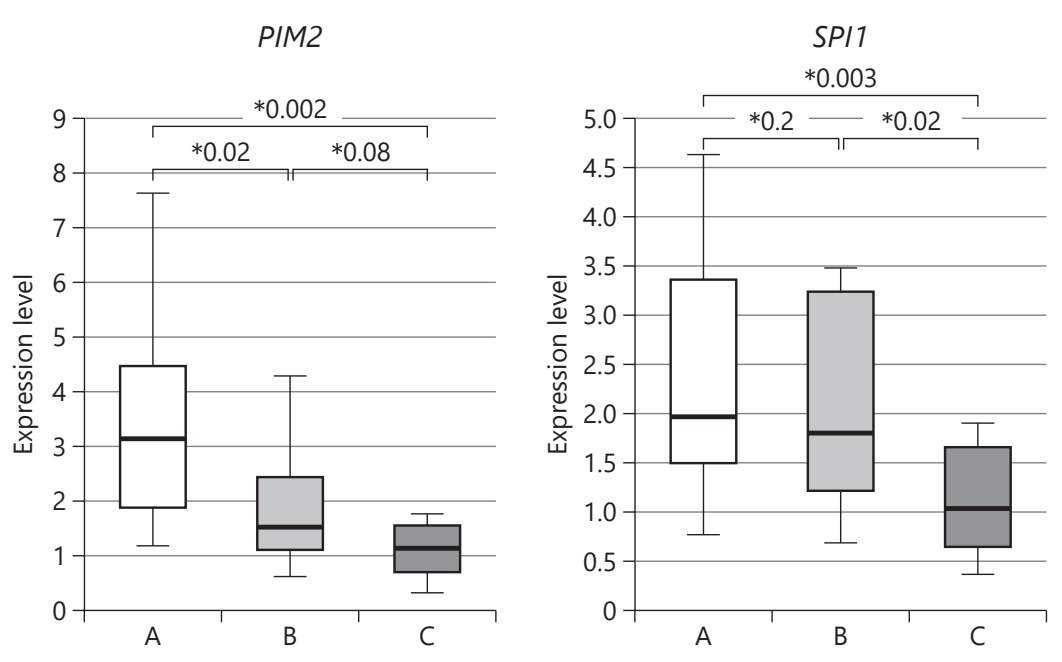

Fig. 2. Real-time PCR analysis of TP53 and $A P C$ expressions in rectal mucosal biopsy specimens. The expression level of each gene normalized to that of GAPDH is presented as a box-and-whisker plot; the central box covers the interquartile range, with the median indicated by the line within the box. The whiskers extend to the maximum and minimum values. $A$, pediatric active UC cases; B, pediatric UC cases in remission; C, pediatric irritable bowel syndrome cases (control). UC, ulcerative colitis; GAP$\mathrm{DH}$, glyceraldehyde 3-phosphate dehydrogenase; PCR, polymerase chain reaction.
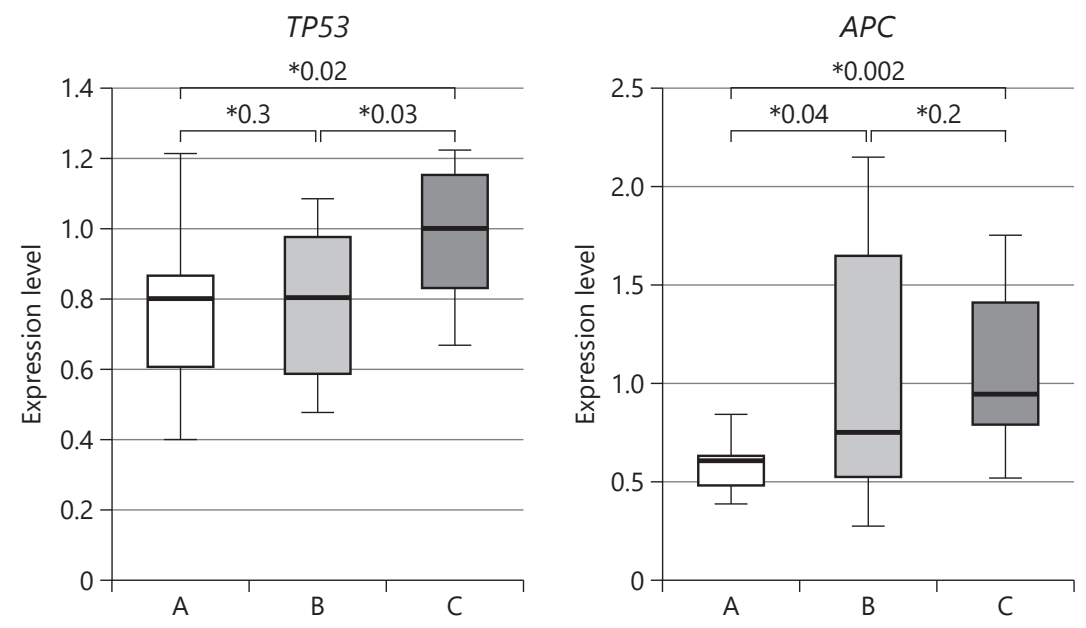

mucosal inflammation from childhood might cause overexpression of cancer-related genes other than those previously reported in colon cancer or adult colitis-associated cancer [8, 14]. PIM2 and SPI1, which have not been previously associated with carcinogenesis of colitis-associated cancer, were expressed at significantly higher levels in pediatric UC patients than in the control group, suggesting their involvement in the development of colon cancer due to inflammatory changes in the gastrointestinal mucosal epithelium.

PIM2 encodes a serine/threonine kinase that regulates cell proliferation signaling and is abundant in hemato- poietic tissues and the gastrointestinal tract. PIM2 is highly expressed in leukemia and colorectal cancer and has been associated with other cancers $[19,20]$. In addition, the PIM2 expression level was reportedly increased in the gastric mucosa of children with Helicobacter $p y$ lori infection and may be involved in gastric cancer [10]. Chronic inflammatory changes in the digestive tract, especially in the mucosal epithelium, were confirmed to increase PIM2 expression, suggesting its potential role in carcinogenesis in the digestive tract. Furthermore, PIM2 expression was restored close to normal levels when mucosal inflammation was improved, and clinical remission 

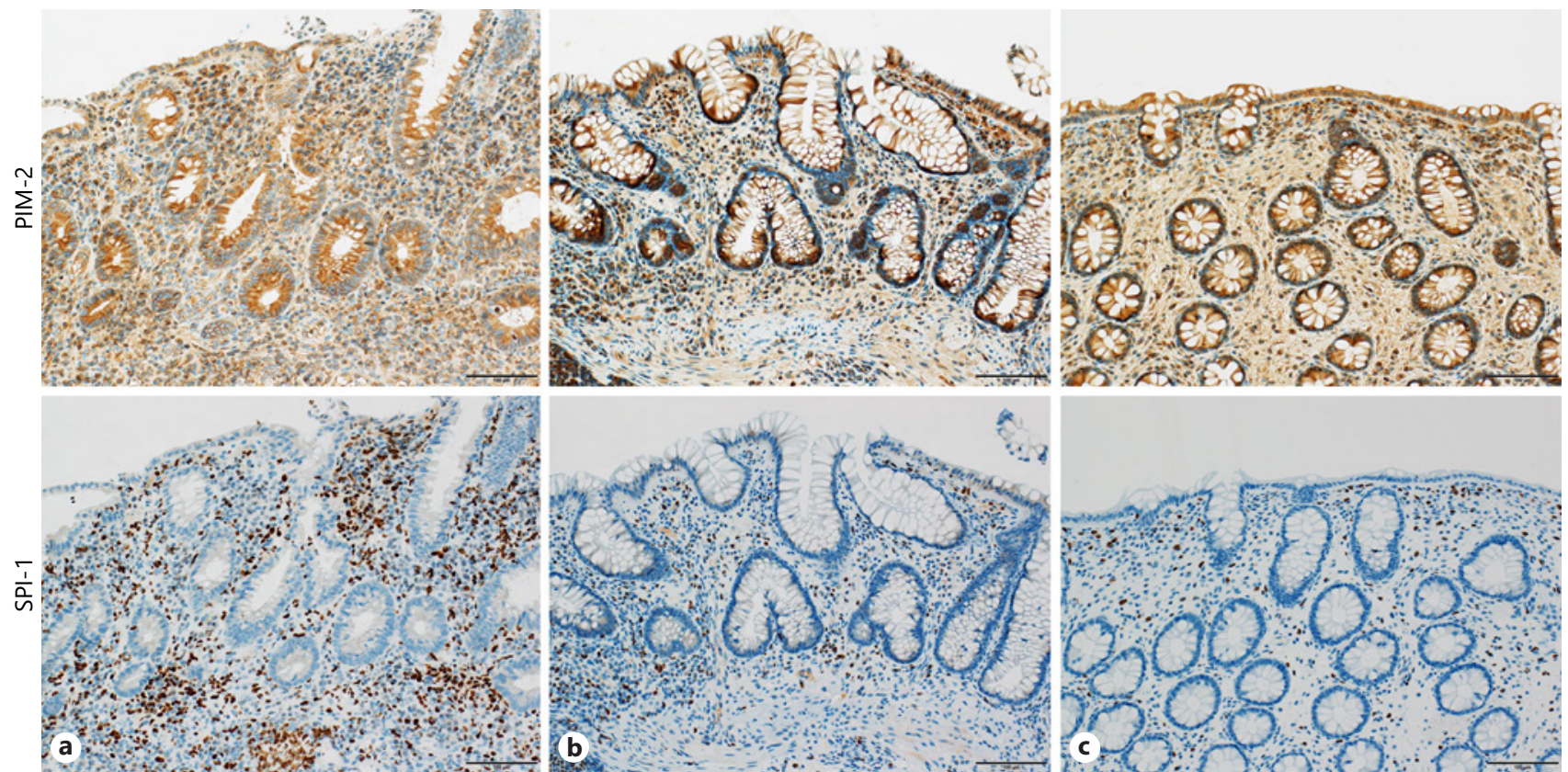

Fig. 3. IHC staining of PIM2 and SPI1 expressions in rectal mucosal biopsy specimens. a Pediatric active cases; b pediatric UC cases in remission; c pediatric irritable bowel syndrome cases (control). Scale bar, $100 \mu \mathrm{m}$. UC, ulcerative colitis; IHC, immunohistochemical.

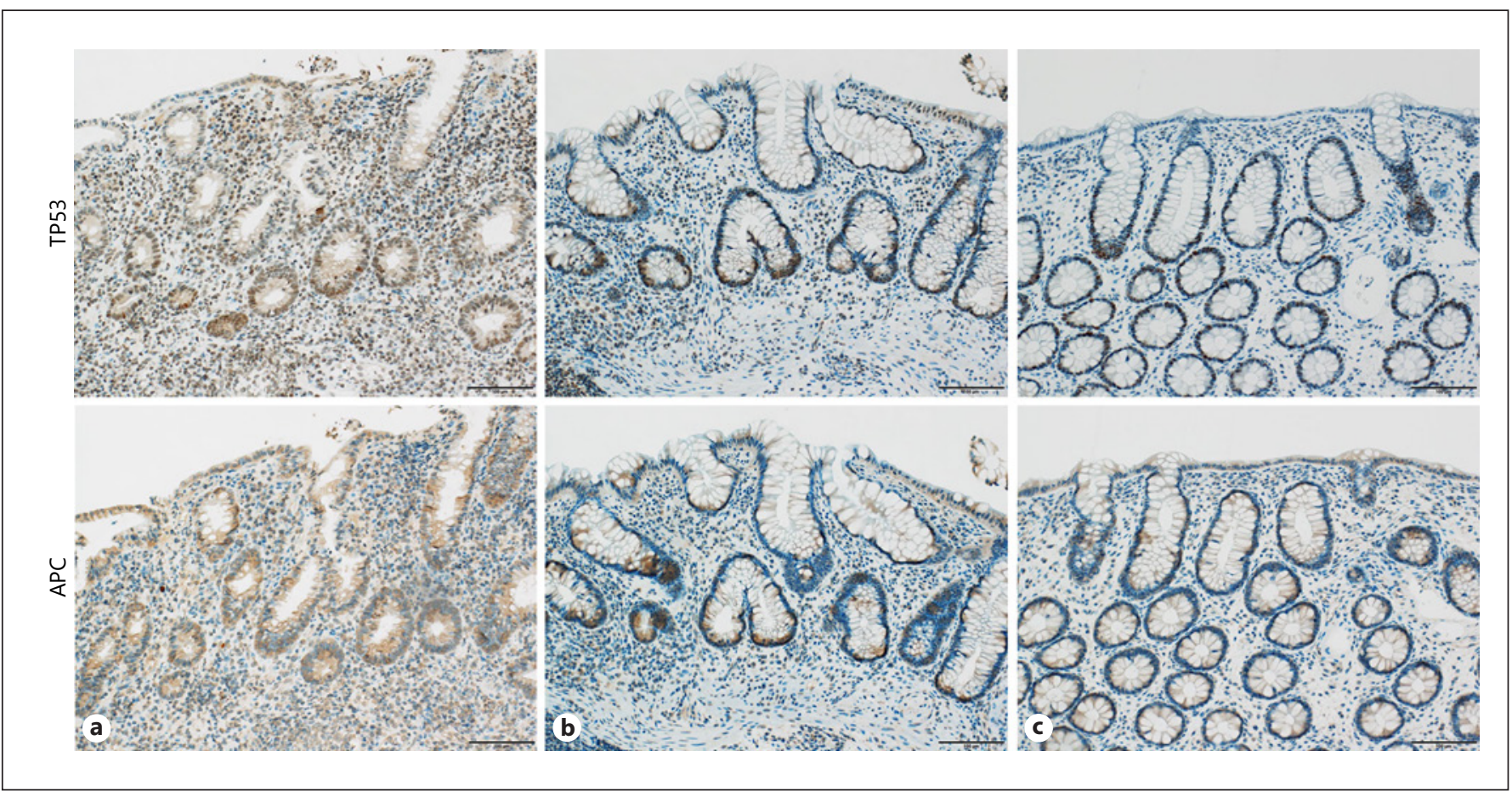

Fig. 4. IHC staining of TP53 and APC expressions in rectal mucosal biopsy specimens. a Pediatric active UC cases; b pediatric UC cases in remission; c pediatric irritable bowel syndrome cases (control). Scale bar, $100 \mu \mathrm{m}$. UC, ulcerative colitis; IHC, immunohistochemical. 
was achieved by proper treatment. These findings suggest that improving chronic inflammation of the gastrointestinal mucosa is an important factor for reducing carcinogenesis risk.

SPI1 encodes a transcription factor that activates the expression of genes involved in cell proliferation in bone marrow [21] and is reportedly a poor prognostic biomarker for childhood T-cell acute lymphoblastic leukemia [22, 23]. SPI1 is also involved in T-cell biology and is reportedly associated with regulatory T cells, UC, colorectal cancer, and colitis-associated cancer [2426]. Therefore, SPI1 might cause colitis-associated cancer by affecting some regulatory $\mathrm{T}$ cells in the mucosa of the digestive tract. In addition, the results of this study demonstrated higher SPI1 expression levels in the active phase of UC when the gastrointestinal mucosa was chronically exposed to inflammatory factors, which was maintained even during the clinical remission period. In addition, IHC analysis revealed increased abundance of SPI1-positive cells among the mucosal epithelial cells, similar to PIM2-positive cells. Generally, it is hypothesized that the number of cancer cells in the epithelium increases because of chronic inflammatory changes, and they then evade selection for apoptosis and gradually become cancerous. Our findings that SPI1 expression remained unchanged throughout UC onset support the suggestion that SPI1 expression might be one of the contributors to the persistently high and increasing risk of colorectal cancer in UC patients over time.

TP53 and APC are both tumor suppressor genes associated with general colorectal cancer without UC [17]. Carcinogenesis suppression is presumed to be impaired if wild-type expression of these genes is reduced or if the gene is mutated. TP53 is often reported in inflamed regions in adult UC patients, as are APC gene mutations [14]. Overexpression of TP53 protein occurs early in UC carcinogenesis, which gradually increases by $90 \%$ in invasive cancer [18]. The cause of TP53 mutations in earlystage UC remains unclear. Analysis of existing genetic abnormalities in the non-neoplastic mucosa of adults at high risk of cancer complications indicated that the carcinogenic risk class could be predicted with $88.7-94.3 \%$ accuracy by analyzing 40 different genes [8]. In the current study, expression levels of TP53 and APC were significantly lower in the UC-active group than in the control group, suggesting that suppression of carcinogenesis was affected even in pediatric UC patients and could lead to carcinogenesis in the future. In addition, both expression and gene mutations of TP53 and APC have been re- ported in colitis-associated cancer [27]. Mutations in $A P C$ occur most often and are estimated to occur in most patients with general colon cancer. However, in colitisassociated cancer, as few as $16 \%$ of colon cancers harbored APC mutations, while as many as $66 \%$ harbored mutations in TP53, further emphasizing the possibility that colitic and general colon cancers develop by different mechanisms. TP53 and APC have also been shown to not only affect general colorectal cancer, but also colitis-associated cancer. Furthermore, a TP53 mutation was also detected in dysplasia, which is a precancerous lesion of colitis-associated cancer and considered already present in the early stage of carcinogenesis from UC $[28,29]$. However, our study suggests that some change might occur between the onset of UC and dysplasia, which could be the initiator of carcinogenesis. These results indicate expression changes of some cancer-related genes even at initial UC onset, and that chronic inflammation from childhood might be involved in the development of colorectal cancer. Recommendations for screening and surveillance of colitis-associated cancer are provided in Europe and the USA [30-32]. Although the Japanese Society of Gastroenterology recommends endoscopy every 1-2 years for patients with colitis and UC, there is no consensus on surveillance of cancer risk in pediatric patients. Based on our findings and those of other studies, monitoring patients and analyzing changes in oncogene expression over time, including additional analysis for patients who have developed cancer, will better enable assessment of cancer risk.

This study had several limitations. Only a small number of patients from a single institution were included for analysis; therefore, the results might be affected by biases in patient backgrounds and regional differences. For this reason, this study should be expanded to include more cases across multiple facilities. In some patients, the time course after UC onset was relatively short, and analysis was performed at an early stage after mucosal inflammation occurred, which might not necessarily constitute chronic inflammatory conditions. Further, the involvement of PIM2 and SPI1 in the oncogenesis of colitis-associated cancer was not elucidated in this study. Moreover, different groups were compared and examined in this study, but analyzing cancer-related gene changes over time would necessitate regular follow-up over an extended period of time. Finally, we only considered pediatric UC patients who had not developed cancer, and neither adult UC patients nor adult patients with colitis-associated cancer were included here. Therefore, confirming whether these gene expres- 
sion changes also occur in adult UC patients or are directly involved in cancer progression requires further study. Already, we are preparing for analysis of adult patients, in collaboration with the Department of Gastroenterology in our hospital and with approval by the Ethics Committee.

\section{Conclusion}

This is the first study to identify cancer-related genes in pediatric UC patients. Furthermore, cancer risk could be stratified according to expression of these genes from initial diagnosis, and indications for preventive colorectal resection could be classified. We also believe that effective therapeutic surveillance should be established from childhood.

\section{Acknowledgments}

The authors thank Ms. Yumiko Sakurai (Department of Pediatric and Adolescent Medicine, Juntendo University Graduate School of Medicine) and Ms. Takako Ikegami, PhD (The Laboratory of Molecular and Biochemical Research, Biomedical Research Core Facilities, Juntendo University Graduate School of Medicine), for their technical support with microarray and real-time PCR analysis. The authors also thank Yuko Kojima, PhD (The Laboratory of Morphology and Image Analysis, Research Support Center, Juntendo University Graduate School of Medicine), for her technical support for IHC analysis.

\section{Statement of Ethics}

This study protocol complied with the ethical guidelines of the Declaration of Helsinki and was approved by the hospital Ethics Committee of Juntendo University Hospital (No. 18-226), and written informed consent was obtained from patients and their legal guardians.

\section{Conflict of Interest Statement}

The authors have no conflicts of interest to declare.

\section{Funding Sources}

There was no research funding on this study.

\section{Author Contributions}

Conception and design of the study, or acquisition of data, or analysis and interpretation of data were performed by Nobuyasu Arai, Takahiro Kudo, Kazuhide Tokita, Reiko Kyodo, Masamichi Sato, and Eri Miyata. Manuscript drafting or revision was done by Kenji Hosoi, Tamaki Ikuse, and Keisuke Jimbo. Yoshikazu Ohtsuka and Toshiaki Shimizu approved the final submitted manuscript.

\section{Data Availability Statement}

The data underlying this article will be shared on reasonable request to the corresponding author.

\section{References}

1 Japan Intractable Disease Information Center. Available from: http://www.nanbyou. or.jp/ Accessed November 11, 2020

2 Herrinton LJ, Liu L, Levin TR, Allison JE, Lewis JD, Velayos F. Incidence and mortality of colorectal adenocarcinoma in persons with inflammatory bowel disease from 1998 to 2010. Gastroenterology. 2012;143:382-9.

3 Eaden JA, Abrams KR, Mayberry JF. The risk of colorectal cancer in ulcerative colitis: a meta-analysis. Gut. 2001;48:526-35.

4 Lutgens MW, van Oijen MG, van der Heijden GJ, Vleggaar FP, Siersema PD, Oldenburg B. Declining risk of colorectal cancer in inflammatory bowel disease: an updated meta-analysis of Population-Based Cohort Studies. Inflamm Bowel Dis. 2013;19:789-99.

5 Shi HY, Chan FK, Leung WK, Li MK, Leung CM, Sze SF, et al. Natural history of elderlyonset ulcerative colitis: results from a territory-wide inflammatory bowel disease registry. J Crohns Colitis. 2016;10(2):176-85.
6 Ekbom A, Helmick C, Zack M, Adami HO Ulcerative colitis and colorectal cancer. A Population-Based Study. N Engl J Med. 1990; 323:1228-33.

7 Olén O, Askling J, Sachs MC, Frumento P, Neovius M, Smedby KE, et al. Childhood onset inflammatory bowel disease and risk of cancer: a Swedish Nationwide Cohort Study 1964-2014. BMJ. 2017;358:j3951.

8 Watanabe T, Kobunai T, Toda E, Kanazawa T, Kazama Y, Tanaka J, et al. Gene expression signature and the prediction of ulcerative colitis-associated colorectal cancer by DNA microarray. Clin Cancer Res. 2007;13:41520.

9 Drossman DA, Hasler WL. Rome IV-functional GI disorders: disorders of gut-brain interaction. Gastroenterology. 2016;150:125761.

10 Obayashi N, Ohtsuka Y, Hosoi K, Ikuse T, Jimbo K, Aoyagi Y, et al. Comparison of gene expression between pediatric and adult gas- tric mucosa with Helicobacter pylori infection. Helicobacter. 2016;21(2):114-23.

11 Kanda Y. Investigation of the freely available easy-to-use software "EZR" for medical statistics. Bone Marrow Transplant. 2013;48:4528.

12 Turner D, Travis SP, Griffiths AM, Ruemmele FM, Levine A, Benchimol EI, et al. Consensus for managing acute severe ulcerative colitis in children: a systematic review and joint statement from ECCO, ESPGHAN, and the Porto IBD working group of ESPGHAN. Am J Gastroenterol. 2011;106: 574-88.

13 Schroeder KW, Tremaine WJ, Ilstrup DM. Coated oral 5-aminosalicylic acid therapy for mildly to moderately active ulcerative colitis. a Randomized Study. N Engl J Med. 1987;317: 1625-9.

14 Ullman TA, Itzkowitz SH. Intestinal inflammation and cancer. Gastroenterology. 2011; 140:1807-16 
15 Vogelstein B, Fearon ER, Hamilton SR, Kern SE, Preisinger AC, Leppert M, et al. Genetic alterations during colorectal-tumor development. N Engl J Med. 1988;319:525-32.

16 Morson BC, Pang LS. Rectal biopsy as an aid to cancer control in ulcerative colitis. Gut. 1967;8:423-34.

17 Takami K, Yana I, Kurahashi H, Nishisho I. Multistep carcinogenesis in colorectal cancers. Southeast Asian J Trop Med Public Health. 1995;26 Suppl 1:190-6.

18 Tanaka H, Fukui H, Fujii S, Sekikawa A, Yamagishi $\mathrm{H}$, Ichikawa $\mathrm{K}$, et al. Immunohistochemical analysis of REG Ia expression in ulcerative colitis-associated neoplastic lesions. Digestion. 2011;83:204-9.

19 Zhang XH, Yu HL, Wang FJ, Han YL, Yang WL. Pim-2 modulates aerobic glycolysis and energy production during the development of colorectal tumors. Int J Med Sci. 2015; 12:48793.

20 Baytel D, Shalom S, Madgar I, Weissenberg R, Don J. The human Pim-2 proto-oncogene and its testicular expression. Biochim Biophys Acta. 1998;1442:274-85.
21 Scott EW, Simon MC, Anastasi J, Singh H. Requirement of transcription factor PU.1 in the development of multiple hematopoietic lineages. Science. 1994;265:1573-7.

22 Takita J. Genetic basis of pediatric T-cell acute lymphoblastic leukemia and its clinical impact. Rinsho Ketsueki. 2018;59:953-9.

23 Özdemir İ, Pınarlı FG, Pınarlı FA, Aksaka FNB, Okur A, Uyar Göçün P, et al. Epigenetic silencing of the tumor suppressor genes SPI1, PRDX2, KLF4, DLEC1, and DAPK1 in childhood and adolescent lymphomas. Pediatr $\mathrm{He}-$ matol Oncol. 2018;35:131-44.

24 Salem M, Wallace C, Velegraki M, Li A, AnsaAddo E, Metelli A, et al. GARP dampens cancer immunity by sustaining function and accumulation of regulatory $\mathrm{T}$ cells in the colon. Cancer Res. 2019;79:1178-90.

25 Iboshi Y, Nakamura K, Fukaura K, Iwasa T, Ogino H, Sumida Y, et al. Erratum to: "Increased IL-17A/IL-17F expression ratio represents the key mucosal $\mathrm{T}$ helper/regulatory cell-related gene signature paralleling disease activity in ulcerative colitis". J Gastroenterol. 2017;52:396-26.

26 Kryczek I, Wu K, Zhao E, Wei S, Vatan L, Szeliga $\mathrm{W}$, et al. IL-17+ regulatory T cells in the microenvironments of chronic inflammation and cancer. J Immunol. 2011;186:4388-95.
27 Fujita M, Matsubara N, Matsuda I, Maejima $\mathrm{K}$, Oosawa A, Yamano T, et al. Genomic landscape of colitis-associated cancer indicates the impact of chronic inflammation and its stratification by mutations in the Wnt signaling. Oncotarget. 2017;9:969-81.

28 Brentnall TA, Crispin DA, Rabinovitch PS, Haggitt RC, Rubin CE, Stevens AC, et al. Mutations in the p53 gene: an early marker of neoplastic progression in ulcerative colitis. Gastroenterology. 1994;107:369-78.

29 Burmer GC, Rabinovitch PS, Haggitt RC, Crispin DA, Brentnall TA, Kolli VR, et al. Neoplastic progression in ulcerative colitis: Histology, DNA content, and loss of a p53 allele. Gastroenterology. 1992;103:1602-10.

30 Sengupta N, Yee E, Feuerstein JD. Colorectal cancer screening in inflammatory bowel disease. Dig Dis Sci. 2016;61:980-9.

31 Clarke WT, Feuerstein JD. Updates in colorectal cancer screening in inflammatory bowel disease. Curr Opin Gastroenterol. 2018;34:208-16.

32 Clarke WT, Feuerstein JD. Colorectal cancer surveillance in inflammatory bowel disease: practice guidelines and recent developments. World J Gastroenterol. 2019;25:4148-57. 